\title{
Schnecken unter Stress
}

\section{Gastropoden als Modelle in Ökophysiologie und Ökotoxikologie}

\author{
Rita Triebskorn
}

Eingegangen: 27 November 2008/Akzeptiert: 23. Februar 2009/ Online veröffentlicht: 20. März 2009

(C) Springer-Verlag 2009

Zusammenfassung Hintergrund Schnecken sind in der ökotoxikologischen und ökophysiologischen Forschung als Zielorganismen für Molluskizide ebenso wie als Nicht-Zielorganismen für Umweltchemikalien und andere Umweltstressoren von Bedeutung. In beiden Fällen werden Biomarker auf verschiedenen biologischen Ebenen untersucht, um zu verstehen, welche Mechanismen die Tiere dazu befähigen, ungünstige Lebensbedingungen zu bewältigen oder gar für sich zu nutzen.

Schwerpunkte Der Schwerpunkt des Artikels liegt auf der Einordnung ökotoxikologischer und ökophysiologischer Arbeiten der Autorin in den Kontext des aktuellen Wissenstandes zu diesem Themenfeld. Hierbei werden neben Reaktionen von Schnecken auf zellulärer Ebene auch biochemische Biomarker (Stressproteine, Metallothioneine, Stoffwechselenzyme) berücksichtigt.

Schlussfolgerungen Die Ausführungen machen deutlich, dass Gastropoden als bedeutende Glieder terrestrischer und aquatischer Nahrungsketten geeignete und sensitive Organismen sind, um Boden- und Gewässerbelastungen $\mathrm{zu}$ beschreiben und zu bewerten. Biomarkerreaktionen bereichern hierbei diesen Forschungszweig insofern, als sie als sensitive Messinstrumente nicht nur Messdaten für die

Meinem Doktorvater, Herrn Prof. Dr. Dr.h. c. Volker Storch gewidmet als Dank für den Nährboden und die Motivation zum Wachsen.

Herausgeber: Henner Hollert · Thomas Braunbeck

R. Triebskorn $(\square)$

Steinbeis-Transferzentrum für Ökotoxikologie und Ökophysiologie,

Blumenstr. 13, 72108 Rottenburg, Deutschland

E-Mail:stz.oekotox@gmx.de

und

Physiologische Ökologie der Tiere,

Universität Tübingen,

Konrad-Adenauerstr. 20, 72072 Tübingen, Deutschland

E-Mail: rita.triebskorn@uni-tuebingen.de angewandte Umweltforschung liefern, sondern gleichzeitig auch wichtige grundlegende Erkenntnisse zur Erklärung funktioneller Zusammenhänge zwischen Stressoren und organismischen Reaktionen auf unterschiedlichen biologischen Ebenen bereitstellen können.

Empfehlungen und Perspektiven Während für terrestrische Ökosysteme ein standardisierter Test mit der Weinbergschnecke zur Verfügung steht, um Chemikalienbelastungen in Böden zu bewerten (ISO 15952), sollte die Sensitivität limnischer Gastropoden z.B. für hormonell wirkende Chemikalien für die Risikobewertung auch in aquatischen Systemen genutzt werden, indem bereits zur Verfügung stehende Testsysteme weiter entwickelt und als Standardtests akzeptiert werden.

Schlüsselwörter Biomarker · Hitzeresistenz Molluskizide · Organische Schadstoffe · Pflanzenschutz · Schnecken $\cdot$ Schwermetalle

\section{Snails under stress - gastropods as models in ecophysiology and ecotoxicology}

Abstract Background In ecophysiology and ecotoxicology, gastropods are important both as target organisms for molluscicides and non-target organisms for environmental pollutants or other environmental stressors. With respect to both aspects, biomarkers are investigated at different levels of biological organization in order to understand mechanisms which enable gastropods to cope with or even to benefit from unfavourable environmental conditions.

Main topics The paper focuses on the ecotoxicological and ecophysiological work of the author on gastropods which will be reviewed in the context of the state of knowledge in this field of research. In addition to cellular aspects in biomarker research, also biochemical responses of snails to 
environmental stress (stress proteins, metallothioneins, and metabolic enzymes) will be addressed.

Conclusions The paper highlights the suitability of terrestrial and aquatic gastropods as sensitive indicators of environmental stress induced by chemicals or other non-chemical factors. Biomarker studies have been shown not only to be applicable in environmental risk assessment but also to provide fundamental and background knowledge necessary to understand correlations of responses at different levels of biological organization.

Recommendations and perspectives A standardized toxicity test with the grapevine snail (ISO 15952) has been established for toxicity assessment in terrestrial habitats. However, freshwater gastropods display a high sensitivity as well, e. g. to endocrine disrupters, and should be incorporated into future standardized assays for aquatic toxicity testing on the basis of existing knowledge.

Keywords Biomarkers ' Gastropds · Heat tolerance · Heavy metals $\cdot$ Molluscicides $\cdot$ Organic chemicals · Pest control $\cdot$ Slugs $\cdot$ Snails

\section{Hintergrund}

Als artenreichste und biologisch vielseitigste Gruppe der Mollusken haben die Schnecken mit 50.000-120.000 beschriebenen bzw. 70.000-200.000 geschätzten Arten (Chapman 2006) erfolgreich marine, limnische und terrestrische Lebensräume besiedelt. Man findet ihre Vertreter in tropischen und kalten Meeren, im fließenden und stehenden Süßwasser und auf den Höhen des Himalajas ebenso wie in trockenen und heißen Wüstenklimaten (Shachak et al. 1987; Fechter und Falkner 1989; Hartmann und Weipert 2006). Ähnlich wie ihre Lebensweise variiert auch der Körperbau der Schnecken extrem. Die kleinsten Arten sind kaum mit bloßem Auge erkennbar, die größte rezente Schnecke, eine Meeresschnecke der Gattung Syrinx, besitzt eine bis zu $70 \mathrm{~cm}$ hohe Schale und wiegt bis zu $13 \mathrm{~kg}$. Schalenlosigkeit ist ebenso verbreitet wie der Besitz einer mehr oder weniger aufwändig gestalteten Schale.

Der Mensch hat seit jeher zu Schnecken eine bivalente Einstellung. Einerseits schätzt er sie als Sammel-, Schmuck-, und Dekorationsobjekte, Nahrungsmittel oder, wie früher in Asien und Afrika und noch heute im Hochland von PapuaNeuguinea, als Zahlungsmittel. In Papua-Neuguinea wurde in diesem Zusammenhang 2002 die weltweit erste MuschelBank, die „Tolai Exchange Bank“ eröffnet. Sie wechselt das Muschelgeld, genannt Tolai, das meist aus Ketten verschiedener Cypraea-Arten besteht, in die gängige Währung, den Kina (Glamm 2005). In der Ökologie und Ökophysiologie sind Gastropoden als wichtige Glieder terrestrischer, limnischer und mariner Ökosysteme geschätzt, die es durch
Anpassungen verhaltensphysiologischer, morphologischer, zellulärer und molekularer Mechanismen geschafft haben, selbst extreme Lebensräume zu erobern. Für Ökotoxikologen haben sie in den letzten dreißig Jahren zusehends als Monitororganismen in der Umweltbewertung und -überwachung Bedeutung erlangt. Zunächst wurden sie als „quantitative Indikatoren für Metallbelastungen" vor allem in terrestrischen Systemen eingesetzt, indem deren Fähigkeit, große Mengen an Schwermetallen zu akkumulieren, genutzt wurde, um Metallbelastungen in der Umwelt darzustellen (Berger und Dallinger 1993; Dallinger et al. 2001). Zelluläre und biochemische Mechanismen, die Schnecken dazu befähigen, selbst in stark metallbelasteten Regionen zu überleben, waren in der Folge und sind bis heute in der ökotoxikologischen Forschung von großem Interesse (Köhler et al. 1996; Triebskorn und Köhler 1996; Dallinger 2007). Einen weiteren Durchbruch als ökotoxikologische Modellorganismen erlangten Schnecken dann in Verbindung mit organischen Umweltschadstoffen: Im Zuge des wachsenden Interesses an möglichen Wirkungen endokriner Disruptoren wurden auch in der aquatischen Ökotoxikologie zusehends invertebrate Tiere als Modellorganismen berücksichtigt. Hierbei erwiesen sich prosobranche Schnecken als äußerst sensitiv für Chemikalien, die das Hormonsystem beeinflussen. Erstmals in der Ökotoxikologie konnten im Sinne einer geschlossenen Kausalkette ökosystemare Effekte, in diesem Fall der weltweite Rückgang verschiedener prosobrancher Schneckenarten, mit deren Exposition gegenüber einer bestimmten Chemikalienklasse, den Organozinnverbindungen, ursächlich in Verbindung gebracht werden (z. B. Oehlmann et al. 1994, Horiguchi et al. 1998; Barreiro et al. 2001). Hierbei waren sowohl der Wirkmechanismus als auch die auf Individualebene resultierenden Effekte bekannt. Auf der Basis dieser Erkenntnisse wurde in der EU ab 1989 die Anwendung von Tributylzinn-(TBT)haltigen Lacken für Schiffsanstriche bei Booten mit einer Länge unter $25 \mathrm{~m}$ verboten. In Deutschland dürfen TBT-haltige Schiffsanstriche seit 2003 überhaupt nicht mehr verwendet werden.

Schnecken sind vom Menschen jedoch bei weitem nicht nur geschätzt. Vielfach werden sie als "schleimig“ und „ekelig“ verachtet, als Schädlinge in Gartenbau und Landwirtschaft gehasst und bekämpft sowie als Überträger und Zwischenwirte von Parasiten gefürchtet. Vor diesem Hintergrund ist die Suche nach effektiven und spezifischen Schneckenbekämpfungsmitteln bis heute Thema in Tropenmedizin und Pflanzenschutz (Henderson und Triebskorn 2002). Im Zuge der Herstellung neuer oder der Verbesserung alter Molluskizide sind Kenntnisse zu Wirkmechanismen von Molluskiziden von Interesse. Ähnlich wie in der Ökotoxikologie werden hierbei zelluläre und biochemische Reaktionen der Tiere untersucht, die als Abwehrmechanismen die Effektivität der Pestizide einschränken oder gar verhindern können. Diese suborganismischen Reaktionen, in der Öko- 
toxikologie auch Biomarker genannt (Köhler und Triebskorn 2004; Triebskorn 2005), werden demnach sowohl in der Ökotoxikologie wie auch in der Phytomedizin als Werkzeuge genutzt, um Reaktionsmöglichkeiten von Schnecken als Ziel- oder Nichtzielorganismen für Chemikalien zu verstehen (Triebskorn et al. 1991, 1996; Triebskorn und Köhler 2003)

In diesem Artikel sollen ausgewählte Bereiche der ökophysiologischen und ökotoxikologischen Forschung mit Schnecken vorgestellt werden, wobei der Schwerpunkt auf Arbeiten der Autorin gelegt wird, die in den Kontext der jeweiligen relevanten Literatur gestellt werden sollen. Ein sehr ausführlicher Übersichtsartikel zur Verwendung von Mollusken als Bioindikatoren in der Ökotoxikologie (Oehlmann und Schulte-Oehlmann 2002) wird als Parallelliteratur empfohlen.

\section{Schnecken als Ziel-Organismen für Molluskizide}

Zielobjekte für Molluskizide sind Gastropoden einerseits als Zwischenwirte von Parasiten und andererseits als Schädlinge in der Landwirtschaft. Im Folgenden kann allerdings nur der zweite Aspekt Berücksichtigung finden.

Schon in der Antike waren Schnecken als Pflanzenschädlinge ein Thema. So findet man in einem Gebet des Märtyrers Trypho von Lampsacus aus dem 10. Jahrhundert, das im Rahmen von Exorzismus-ähnlichen Riten eingesetzt wurde, Folgendes: „O ye, Caterpillars, Worms, Beetles, Locusta, Grasshoppers, Wooly-Bears, Wireworms ... Snails, Earwigs and all other creatures that cling to and wither the fruit of the grape and all other herbs, I charge you by the many-eyed Cherubim, and by the six-winged Seraphim ... and by the holy Angels and all the powers, hurt not the vines nor the land nor the fruit of the trees nor the vegetables ... but depart into the wild mountains, into the unfruitful woods ... ." (Harmer 1895).

Als Schadschnecken in Landwirtschaft sowie Haus- und Kleingarten sind heute weltweit vor allem Nacktschnecken der Gattungen Arion (Arion lusitanicus, Arion distinctus/ hortensis) und Deroceras (Deroceras reticulatum) von Bedeutung. Hinzu kommen mit geringerer Bedeutung terrestrische Gehäuseschnecken der Gattungen Cepaea (v.a. Cepaea hortensis), Helix (Helix aspersa) und Theba (Theba pisana), letztere vor allem im Mittelmeerraum, in den USA und in Australien als Schädling im Weinbau. Vertreter der Gattungen Pila bzw. Pomacea (Apfelschnecken) sind in Asien als Schädlinge im Reisanbau relevant.

Neben zahlreichen Möglichkeiten, die der biologische Pflanzenschutz bietet (z. B. Allgaier und Albert 2008), ist die chemische Bekämpfung von Schadschnecken immer noch die favorisierte Methode, um Pflanzen vor Schneckenfraß zu schützen (Henderson und Triebskorn 2002).
Zur chemischen Schneckenbekämpfung werden derzeit schwerpunktmäßig drei Wirkstoffe eingesetzt: Methiocarb, Metaldehyd und Eisen-III-Phosphat. In der Vergangenheit auf ihre mögliche Eignung als Molluskizide überprüft wurden ein weiterer Carbamatwirkstoff, das Cloethocarb, sowie zwei Eisenverbindungen, Eisenbutan und Eisenoctan. Während der Wirkmechanismus von Methiocarb (im Handel unter dem Namen Mesurol erhältlich) und Cloethocarb als Neurotoxine bzw. Cholinesterasehemmer bekannt war, war für Metaldehyd und die Eisenverbindungen (EisenIII-Phosphat, heute im Handel unter dem Namen Ferramol erhältlich) lange Zeit fraglich, worauf die Wirkung dieser Stoffe bei Schnecken beruht. Auch zur Effektivität von Cloethocarb im Vergleich zu Methiocarb war wenig bekannt.

Im Rahmen der von der Autorin durchgeführten Untersuchungen zu diesem Themenkomplex konnte zunächst mittels Mikro-Röntgentechnik, Autoradiographie, Atomabsorptionsspektralphotometrie sowie Filterelektronenmikroskopie die Passage einzelner Molluskizide im Körper von Nacktschnecken verfolgt und auf zellulärer Ebene Angriffspunkte für die einzelnen Wirkstoffe dargestellt werden (Triebskorn et al. 1990, 1999; Triebskorn und Florschütz 1993; Triebskorn und Köhler 2003). Für Cloethocarb zeigten diese Studien, dass der Wirkstoff den Nahrungstransport im Darm verlangsamt sowie selbst im Kropf resorbiert und über die Hämolymphe zur Mitteldarmdrüse transportiert wird. Dort wird er schwerpunktmäßig in einem Zelltypus, der Kalkzelle (syn. Basophile Zelle bei aquatischen Mollusken) nachgewiesen. Da dieser Zelltyp an Biotransformationsprozessen beteiligt ist, ist davon auszugehen, dass hier auch Metabolisierungs- bzw. Entgiftungsprozesse stattfinden, die die Effektivität dieses Stoffes mindern. Im Vergleich zu Mesurol ist Cloethocarb deutlich weniger toxisch für Schnecken, obgleich es zu zellulären Schäden in verschiedenen Organen der belasteten Tiere führt (Triebskorn und Künast 1990).

Metaldehyd kann vor allem in Schleimzellen des Darmtraktes und der Haut, aber auch in der Mitteldarmdrüse nachgewiesen werden. An diesen Stellen entfaltet dieses Molluskizid dann auch seine toxische Wirkung. Nach zunächst verstärkter Schleimbildung kommt es, auch bei niedriger Temperatur und hoher Luftfeuchtigkeit, zur Zerstörung der Schleimzellen, was den Tod der Tiere zur Folge hat (Triebskorn und Ebert 1989; Triebskorn und Schweizer 1990; Triebskorn et al. 1998). Die Wirkung von Metaldehyd basiert letztlich auf der Beeinflussung des Serotoninstoffwechsels (Triebskorn et al. 1998). Sekundär werden dann Wasserund Ionenhaushalt der Tiere sowie der Energiestoffwechsel gestört. Auf der Basis dieser Untersuchungen konnte die verbreitete Meinung widerlegt werden, Metaldehyd entfalte seine Wirkung nur unter trockenen und warmen Bedingungen.

Während das Carbamat Cloethocarb bei Schnecken vorwiegend in den Kalkzellen der Mitteldarmdrüse nach- 
gewiesen werden konnte, ließen sich Eisenverbindungen (Eisenbutan und Eisenoktan) mit Hilfe der Filterelektronenmikroskopie vor allem in den Resorptionszellen der Mitteldarmdrüse lokalisieren (Triebskorn et al. 1999). Auch Ferramol, das heute handelübliche Produkt auf Eisen-IIIBasis, akkumuliert sehr deutlich in der Mitteldarmdrüse von Schnecken bereits nach 4 h (Triebskorn und Köhler 2003). Anders als Cloethocarb, das vorwiegend in den Kalkzellen bzw. basophilen Zellen angereichert und dort ggf. auch entgiftet wird, werden die Eisenverbindungen in der Mitteldarmdrüse offensichtlich nicht abgebaut, sondern zerstören innerhalb von $16 \mathrm{~h}$ das Epithel dieses Organs. Als Folge bricht auch die zelluläre Stressantwort in Form der Hitzeschockproteine bei diesen Tieren zusammen (Triebskorn und Köhler 2003).

Neben den genannten drei Wirkstoffklassen gibt es auch in jüngster Zeit immer wieder Versuche, Molluskizide auf Alternativbasis zu entwickeln. Beispielsweise wurde die Verwendung von Koffein oder Knoblauchextrakten als molluskizide Inhaltsstoffe für Schneckenköder diskutiert (Hollingsworth et al. 2002; Schuder et al. 2003). Bis heute wurde allerdings noch keine dieser Ideen industriell umgesetzt.

Insgesamt hat sich gezeigt, dass histologische und elektronenmikroskopische Untersuchungen verschiedener Gewebe von Schnecken in Verbindung mit Stoff- bzw. Elementnachweisen sehr gut geeignet sind, Aufnahmewege, Reaktionsmöglichkeiten der Tiere sowie schädigende Wirkungen zu verstehen. Auf ultrastruktureller Ebene haben sich hierbei Antworten des endoplasmatischen Reticulums vor allem in den Kalkzellen der Mitteldarmdrüse und in den Schleimzellen des Mantels und der Sohle als sehr informativ erwiesen, um den zellulären Reaktionsgrad in den betreffenden Geweben abschätzen zu können (Triebskorn und Köhler 1992).

\section{Schnecken als Nichtziel-Organismen für Umweltchemikalien}

\subsection{Metalle}

Bereits in den 80er-Jahren des letzten Jahrhunderts wurde der Tatsache, dass Landschnecken als Akkumulationsindikatoren für Schwermetalle genutzt werden können, große Beachtung geschenkt (z.B. Coughtrey und Martin 1977; Russel et al. 1981; Dallinger und Wieser 1984). Gomot-De Vaufleury und Pihan (2002) konnten zeigen, dass Metalle von Schnecken sowohl über die Nahrung als auch über die Sohle aufgenommen werden. In den Resorptions-, Exkretions- und Kalkzellen der Mitteldarmdrüse (syn. Hepatopankreas; Abb. 1) werden diese dann in Form von Granula (syn. Sphaeriten) immobilisiert. Hierbei unterscheidet man in Abhängigkeit von den gebundenen Metallarten unterschiedliche Granulatypen. In den konzentrisch aufgebauten
Typ-A-Granula (Abb. 2) werden vor allem Calcium, Mangan, Eisen, Kobalt und Zink, in den eher unregelmäßig geformten Typ-B-Granula Cadmium, Kupfer und Quecksilber gespeichert (Taylor 1995). Recio et al. (1988) und Marigomez et al. (1997) konnten in der Mitteldarmdrüse von Arion ater dementsprechend auch Zink in den konzentrisch aufgebauten Typ-A-Sphaeriten der Kalkzellen und Quecksilber in Vakuolen der Resorptionszellen nachweisen. In Abb. 3 ist zu erkennen, dass die Typ-A-Sphaeriten in den Kalkzellen der Mitteldarmdrüse der prosobranchen Landschnecke Pomatias elegans sehr viel Calcium speichern und deutlich positiv mit der Nachweismethode nach von Kossa angefärbt werden. Bevor die Immobilisierung von Metallen in Sphaeriten stattfindet, werden Metalle intrazellular an metallbindende Proteine, sog. Metallothioneine gebunden. Dallinger et al. (1997) konnten zeigen, dass Helix pomatia und Arianta arbustorum metallspezifische Isoformen dieser Proteine in Mitteldarmdrüse und Sohle besitzen. In der Mitteldarmdrüse findet man Isoformen, die bevorzugt Cadmium binden (Hispard et al. 2008), in einem bestimmten Zelltypus von Mantel und Sohle (der Rhogozyte oder Porenzelle) solche, die am Kupfermetabolismus beteiligt sind (Dallinger et al. 2005). Jede Isoform ist in der Lage, eine maximale Anzahl bestimmter Metallionen zu binden. So weiß man mittlerweile, dass ein Molekül Cd-Metallothionein in der Mitteldarmdrüse von Schnecken maximal sechs Cadmiumionen binden kann (Chabicovsky et al. 2004). Ist der Metallothioneinpool gesättigt, kann Cadmium nicht mehr entgiftet werden und es kommt zu zellulären Schädigungen (Chabicovsky et al. 2004). Informationen über den Sättigungsgrad von Metallothioneinen mit entsprechenden Metallionen geben demnach Auskunft über mögliche, durch Überlastung des Metallothionein-Schutzsystems entstandene Schadwirkungen bei Schnecken. Vor diesem Hintergrund können Metallothioneine nicht nur als Biomarker, die den Grad der Metallbelastung indizieren (sog. Biomarkers of exposure), sondern auch als Biomarker, die mögliche Effekte in den Organismen voraussagen (sog. Biomarkers of effect) eingesetzt werden. Zelluläre Effekte nach Metallbelastung von Nacktschnecken wurden z.B. von Triebskorn und Köhler (1996), Marigomez et al. (1997), Köhler und Triebskorn (1998) und Zaldibar et al. (2007) beschrieben. Quecksilber und Cadmium führten bei Arion ater zur fast vollständigen Reduktion der Resorptionszellen und zur Dominanz von Kalkzellen im Mitteldarmdrüsenepithel (Marigomez et al. 1997; Zaldibar et al. 2007). In den Kalkzellen wurde in Reaktion auf Metallbelastung bei Arion lusitanicus eine besondere Form des endoplasmatischen Reticulums, das sog. Mannosom, vermehrt nachgewiesen. Assoziiert mit diesem Organell findet man das Enzym D-Mannitol-Oxidase, das als eine Art „Stressenzym“ bei Schnecken fungieren soll (Knigge et al. 2002). Als weitere Effektmarker für Metallbelastungen können der Lumenradius der Tubuli, der sich unter Belastung in der Regel vergrößert, sowie die Epithelhöhe, die 
unter Belastung meist abnimmt, genutzt werden (z. B. Marigomez et al. 1997). Bei Deroceras reticulatum verursachten Blei und Cadmium sehr starke zelluläre Schäden in der Mitteldarmdrüse (Triebskorn und Köhler 1996). Blei, das nicht an Metallothioneine gebunden und in nur geringem Ausmaß in Geweben angereichert wird, führte hierbei in sehr viel geringeren Konzentrationen zur Überlastung der molekularen Stressantwort in Form von Stressproteininduktion als Cadmium, das an Metallothioneine gebunden werden kann und in Folge dessen auch deutlich stärker akkumuliert (Triebskorn und Köhler 2003). Die Relevanz dieser molekularen Stressantwort für zu erwartende Effekte auf der Populationsebene (Reproduktion und Überlebensrate) wurde von Köhler et al. (1998) gezeigt. Coeurdassier et al. (2002) fanden bei der Weinbergschnecke eine direkte Korrelation zwischen der Konzentration an Metallen in verschiedenen Geweben und der Hemmung des Wachstums der Tiere. Vor diesem Hintergrund wurde von den Autoren ein standardisierter Test entwickelt, der eingesetzt wird, um Chemikalienbelastungen in Böden zu bewerten (ISO 15952 2006). Dieser Test ist besonders zur Beurteilung von Metallbelastungen geeignet, wohingegen er sich als relativ unsensitiv für polyaromatische Kohlenwasserstoffe erwies (Sverdrup et al. 2006).

Für aquatische Schnecken liegen insgesamt sehr viel weniger Daten zur Metalltoxizität vor als für terrestrische Gastropoden. Khangarot und Ray (1988) zeigten, dass Lymnaea luteola sehr sensitiv auf Quecksilber, Kupfer und Cadmium reagiert. Auch Gomot (1998) konnte negative Effekte von Cadmium hinsichtlich Eiproduktion, Schlupf und Embryonalentwicklung bei Lymnaea stagnalis nachweisen. Auf der Basis ähnlicher Messparameter wurde von Schirling et al. (2006) ein Embryotest mit der Apfelschnecke Marisa cornuarietis entwickelt, der, ähnlich wie der in der Ökotoxikologie etablierte Embryotest mit dem Zebrabärbling, für die Bewertung der Toxizität von Chemikalien, Chemikaliengemischen oder auch Wasser- und Sedimentproben eingesetzt

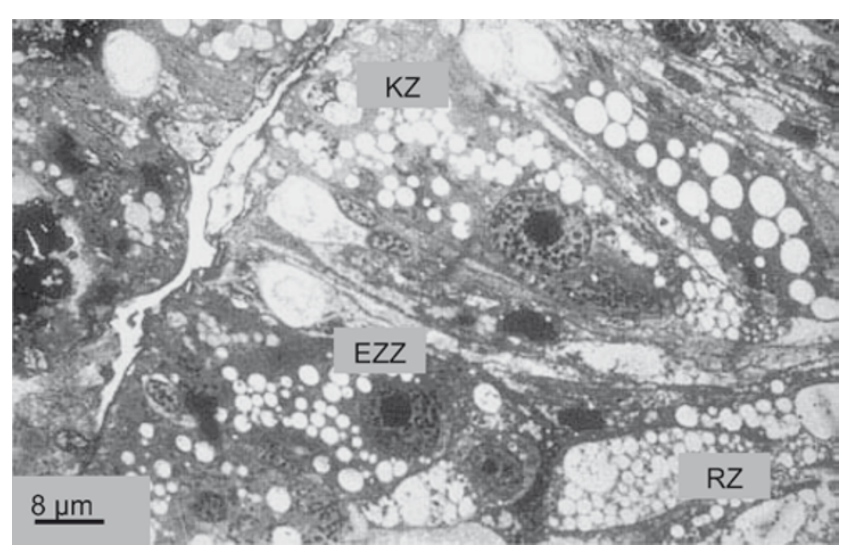

Abb. 1 Epithel der Mitteldarmdrüse von Pomatias elegans. $R Z$ Resorptionszelle, $K Z$ Kalkzelle, EZ Exkretionszelle

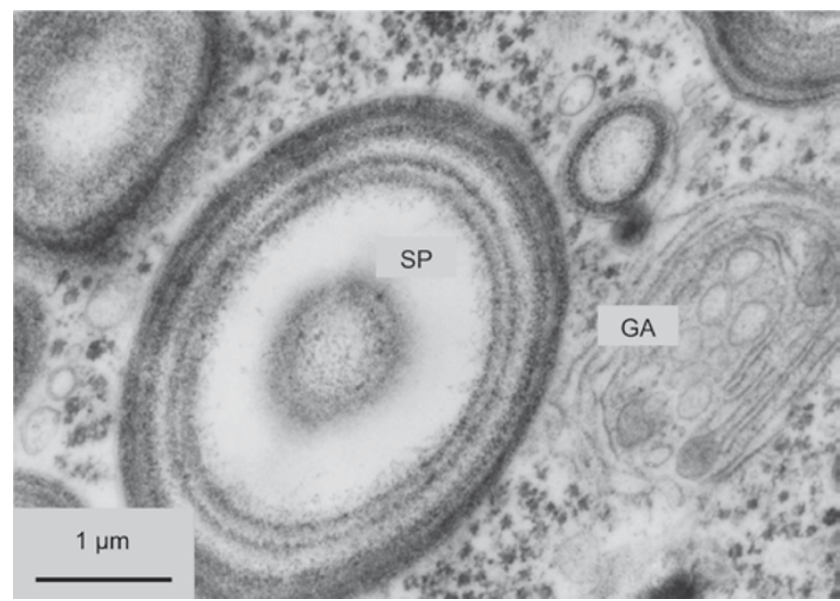

Abb. 2 Typ-A-Sphaerit in einer Kalkzelle der Mitteldarmdrüse von Pomatias elegans. SP Typ-A-Sphaerit, GA Golgiapparat

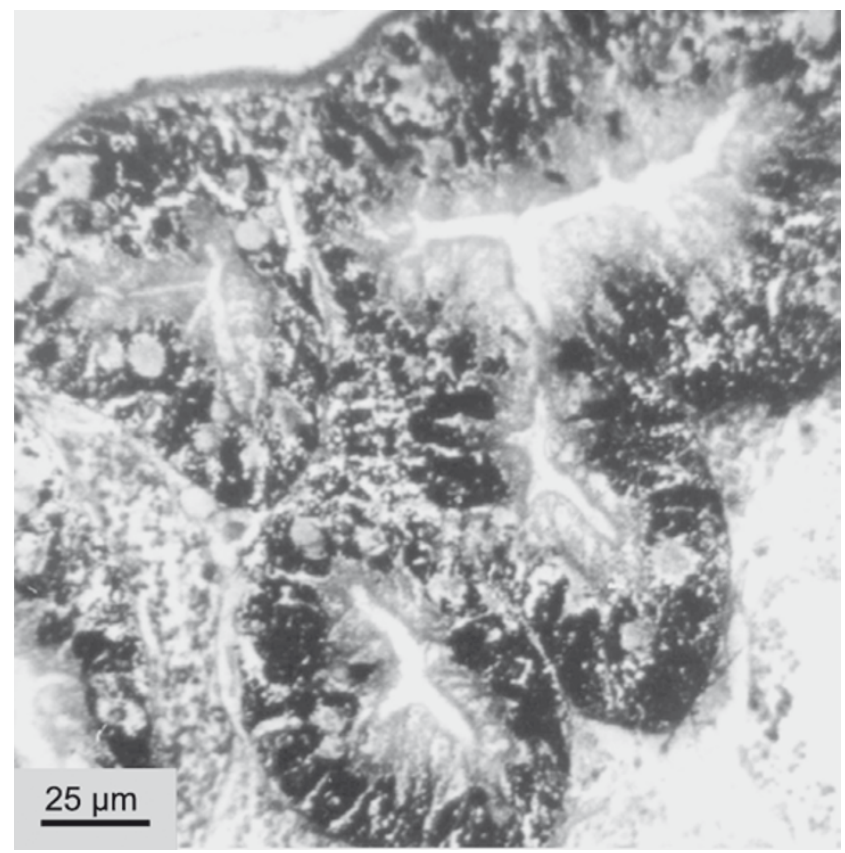

Abb. 3 Calciumnachweis nach von Kossa in der Mitteldarmdrüse von Pomatias elegan

werden kann. Sawasdee und Köhler (2008) untersuchten mit diesem Test die Embryotoxizität von Nickel, Blei und Zink und konnten zeigen, dass der Test ungefähr zehnmal sensitiver für Metallbelastungen ist als der Zebrabärblingstest. Empfindlichste Endpunkte waren hierbei die Augenund Tentakelentwicklung sowie die Schlupfrate. Osterauer et al. (2008) beobachteten in dem gleichen Testsystem die Hemmung der Schalenbildung von Marisa cornuarietis durch ein Platingruppenelement. Reed-Judkins et al. (1997) untersuchten schließlich in Langzeittests chronische Effekte von Kupfer und Zink bei der Schnecke Leptoxis praerosa und kamen zu dem Schluss, dass die Qualitätsvorgaben der 
US EPA (Environmental Protection Agency), die auf Kurzzeittests beruhen, für diese beiden Metalle das Risiko für aquatische Invertebraten unterschätzen.

Abschließend soll noch erwähnt werden, dass das Wissen um die Toxizität von Metallen für Schnecken lange Zeit genutzt wurde, um diese, vor allem als Zwischenwirte von Parasiten, aber auch in der Landwirtschaft im Sinne von Ziel-Organismen für Chemikalien zu bekämpfen. Eingesetzt wurden hierbei vor allem Kupfersalze, aber auch Aluminiumsalze oder Eisenverbindungen fanden Verwendung (Henderson und Triebskorn 2002). Auf Eisenphosphatbasis beruht heute eines der bedeutendsten handelsüblichen Molluskizide, das Ferramol (s. Abschn. 2).

\subsection{Organika}

Aquatische prosobranche Schnecken haben sich als extrem sensitiv für hormonell wirksame Umweltchemikalien erwiesen (Oehlmann und Schulte-Oehlmann 2003). Aufgrund einer relativ labilen Geschlechtsdifferenzierung zeigen sie in Abhängigkeit von Konzentration und Wirkmechanismus der Testsubstanzen verschiedene Symptome, die als Imposex, Intersex oder Superfeminisierung bekannt sind.

Vor diesem Hintergrund musste die lange Zeit weit verbreitete Meinung revidiert werden, invertebrate Tiere hätten keine Vertebraten-ähnlichen Rezeptoren, die von Umwelthormonen besetzt werden könnten, und würden demnach auch nicht mit spezifischen Antworten reagieren, die mit endokriner Disruption im Zusammenhang stehen (Köhler et al. 2007).

Als Imposex wird die Bildung zusätzlicher männlicher Geschlechtsmerkmale (Penis und/oder Vas deferens) bei weiblichen gonochoristischen prosobranchen Schnecken bezeichnet. Im fortgeschrittenen Stadium führt dies zur Infertilität der Weibchen. Der Effekt ist spezifisch für die Wirkung von Tributylzinnverbindungen (TBT), tritt in sehr geringen Konzentrationen auf und wurde bis heute weltweit bei mehr als 150 prosobranchen Schneckenarten beobachtet (Oehlmann und Schulte-Oehlmann 2003). Als Intersex bezeichnet man ein verwandtes Phänomen, bei dem weibliche Geschlechtsmerkmale durch männliche Anteile ersetzt werden, wodurch es zur Sterilität der Weibchen kommt. Intersex tritt vor allem bei Littoriniden nach Exposition gegenüber höheren TBT-Konzentrationen auf (Oehlmann et al. 1998). Für beide durch TBT hervorgerufenen Effekte ist die Kausalkette von (1) der Exposition gegenüber TBT über (2) molekulare, biochemische und morphologische Konsequenzen auf Individualebene bis hin zu (3) ökosystemaren Effekten (extremer Rückgang ganzer Populationen prosobrancher Schneckenarten vorwiegend in Hafennähe) fast komplett geschlossen (Matthiessen und Gibbs 1998; Oehlmann und Schulte-Oehlmann, 2003; Gibbs 2008). Auf molekularer Ebene ist für die Ausprägung von Imposex und Intersex derzeit die kompetitive Hemmung der Cytochrom-
P-450-vermittelten Aromatase der wahrscheinlichste Wirkmechanismus, wodurch es zu erhöhten Testosteronleveln in den Tieren kommt (Matthiessen und Gibbs 1998); es werden jedoch auch konkurrierende Erklärungsmodelle diskutiert (vgl. Übersicht in Oehlmann et al. 2007).

Aufgrund der drastischen Freilandeffekte, die auf TBTBelastungen ursächlich zurückgeführt werden konnten, wurde 1989 in der EU der Einsatz von TBT-haltigen Schiffsanstrichfarben für Schiffe mit einer Länge unter $25 \mathrm{~m}$ verboten. Da die TBT-Belastungen trotz des Verbotes nicht deutlich zurückgingen, legte im Januar 2001 die Bundesregierung veranlasst durch einen Entscheid der Internationalen Seeschifffahrts-Organisation (IMO) der Europäischen Kommission den Entwurf einer nationalen TBT-Verbotsverordnung vor. Durch diese sollte die Abgabe und Verwendung von Schiffsanstrichen, die zinnorganische Verbindungen enthalten, vollständig verboten werden. Im August 2002 setzte schließlich das Bundeskabinett die Richtlinie der Europäischen Union (EU) zum vollständigen TBT-Verbot in nationales Recht um. Schiffslacke auf TBT-Basis sind deshalb heute in Deutschland vollständig verboten.

Neben Imposex und Intersex tritt bei limnischen prosobranchen Schnecken nach Exposition gegenüber östrogenartig wirkenden Chemikalien das Phänomen der „Superweibchen" auf. Hierbei werden zusätzliche weibliche Geschlechtsorgane (z.B. eine zweite Vagina) ausgebildet, die akzessorischen weiblichen Geschlechtsdrüsen werden vergrößert und man findet Eileitermissbildungen. Insgesamt wird die Eiablage stimuliert und die Mortalität bei Weibchen ist erhöht (Schulte-Oehlmann et al. 2001). Dieses Phänomen wurde u. a. für die Apfelschnecke Marisa cornuarietis nach Exposition gegenüber umweltrelevanten Konzentrationen von Bisphenol A (BPA) beschrieben (Schulte-Oehlmann et al. 2001; Oehlmann et al. 2006). Um diese sowie weitere Studien gibt es nach wie vor ausgiebige Diskussionen um Tiermaterial, experimentelles Design, notwendige Voraussetzungen für die Validität der Untersuchungen sowie die statische Auswertung der Daten. In drei Arbeiten von Forbes et al. (2007a, 2007b, 2008) werden die Ergebnisse von Oehlmann et al. $(2000,2006)$ als nicht zutreffend dargestellt. Von Van der Hoeven (2005) wurde schließlich eine Gegendarstellung zu Oehlmann et al. (2000) publiziert, die in das neue Risk Assessment für BPA (EU 2008) eingeflossen ist. Zu diesem nehmen Oehlmann et al. (2008) dann wiederum kritisch Stellung. Die Brisanz der Diskussion macht deutlich, wie heftig im Zusammenhang mit möglichen hormonellen Wirkungen einer wirtschaftlich sehr bedeutsamen Substanz wie Bisphenol A um die Wahrheit gekämpft wird. Anzumerken ist in diesem Zusammenhang auch, dass die Studien von Forbes et al. mit Ko-Autorenschaft von Vertretern der BPA-produzierenden Industrie publiziert und von dieser finanziert wurden. Negative Effekte von Bisphenol A wurden jedoch nicht nur bei Apfelschnecken beobachtet. 
Bei Potamopyrgus antipodarum trat eine vermehrte Bildung von Embryonen während der sexuellen Ruhephase sowohl nach Exposition gegenüber Bisphenol A als auch gegenüber UV-Filtersubstanzen (Inhaltsstoffe in Sonnenschutzmitteln) auf (Schulte-Oehlmann et al. 2001; Schmitt et al. 2008). Der gleiche Effekt war bei dieser Schneckenart festzustellen, wenn die Tiere gegenüber komplex belasteten Sedimenten exponiert wurden, in denen hohe östrogene Potentiale nachzuweisen waren (Mazurová et al. im Druck). Testsysteme, die die Schlammschnecke Lymnaea stagnalis als Modellorganismus zum Nachweis endokriner Effekte nutzen, zeigten bislang eher variable Ergebnisse (Matthiessen 2008).

Negative Einflüsse von Pestiziden auf die Embryonalentwicklung von Marisa cornuarietis konnten Sawasdee und Köhler (im Druck) für Atrazin und Imidacloprid nachweisen. Sensible Endpunkte waren in dieser Studie die Herzschlagrate und der Schlupfzeitpunkt.

Insgesamt gesehen haben die beschriebenen Versuche deutlich gemacht, dass heute verschiedene Testsysteme mit aquatischen Schnecken zur Verfügungen stehen, durch die mögliche endokrine Wirkungen und toxische Effekte von Einzelchemikalien und komplexen Umweltproben nachgewiesen werden können. Die Optimierung dieser Systeme vor allem hinsichtlich Reproduzierbarkeit ist allerdings laut Matthiessen (2008) noch nicht so weit fortgeschritten, dass die Tests zum jetzigen Zeitpunkt problemlos als international standardisierte Testmethoden eingesetzt werden könnten.

Untersuchungen zu Wirkungen von Organika bei terrestrischen Schnecken sind vielfach auf Wirkungen von Schneckenbekämpfungsmitteln ausgerichtet (s.o.). Einzelne Studien betreffen mögliche Effekte von Pestiziden und polyaromatischen Kohlenwasserstoffen (PAHs) bei verschiedenen Schneckenarten. Nebenwirkungen von im Weinbau eingesetzten Pestiziden auf Helix pomatia wurden von Triebskorn et al. (2005) untersucht. Die beiden Testprodukte Sufran (Netzschwefel) und Polyram (Metiram) erwiesen sich hierbei für die Schnecken als unproblematisch. Regoli et al. (2006) setzten Helix aspersa als Monitororganismus an stark befahrenen Straßen ein, um mögliche, durch Luftverschmutzung hervorgerufene Gesundheitsrisiken für den Menschen abzuschätzen. Hierbei konnten sie relativ gute Korrelationen zwischen dem Verkehrsaufkommen, der Steigerung der Aktivität von Biotransformationsenzymen (EROD), Metallothioneingehalte und der Proliferation von Peroxisomen in der Mitteldarmdrüse nachweisen. Laguerre et al. (im Druck) nutzen die Induktion von B-Esterasen bei der Heideschnecke Xeropicta derbentina erfolgreich als Biomarker zum Nachweis von negativen Effekten von Organophosphaten bei dieser Schneckenart. Der standardisierte Schneckentest nach ISO 159522006 hat sich, wie bereits erwähnt, für polyaromatische Kohlenwasserstoffe wenig bewährt (Sverdrup et al. 2006).

\section{Schnecken und Hitzestress}

Die Auseinandersetzung von Landschnecken mit ihrer Umgebungstemperatur ist seit langem Gegenstand der ökophysiologischen Forschung. Physiologische Probleme mit dem Wasserhaushalt und der Nahrungsgrundlage wurden bereits von Schmidt-Nielsen et al. (1971) beschrieben. Auch die große Toleranz von Landschnecken gegenüber hoher Temperatur und Trockenheit ist lange bekannt (z. B. Gebhardt-Dunkel 1953; Asami 1993; Arad et al. 1993). Mediterrane Landschnecken sind, bedingt durch starke Sonneneinstrahlung in offenen Habitaten, extrem hohen Temperaturen ausgesetzt. Heideschnecken, zu denen Vertreter der Familien Hygromiidae und Helicidae zusammengefasst werden, zeigen unter diesen Extrembedingungen das Verhalten, dass sie an vertikal stehenden Objekten (Gräser, Pfähle etc.) nach oben kriechen, um kühlere Bedingungen als am Boden zu haben (Cowie 1985). Dort verschließen sie die Schalenmündung mit einer Haut aus Schleim, mit der sie sich auch am Untergrund festkleben. Dieses Verhalten zeigen vorwiegend adulte Tiere, da Juvenile anfälliger gegenüber Austrocknung sind und deshalb Bereiche höherer Luftfeuchtigkeit in der Nähe des Bodens aufsuchen (Arad und Avivi 1998). Ein Aufenthalt direkt auf dem Boden ist für die Tiere letal (Kempster und Charwa 2003). Obwohl dieses Phänomen seit langem bekannt ist, fehlen detaillierte Untersuchungen $\mathrm{zu}$ physiologischen Voraussetzungen bei diesen Tieren für die beschriebenen Verhaltensweisen. Bekannt ist, dass unterschiedliche Arten und sogar unterschiedliche Populationen der gleichen Art unterschiedliche Temperaturmaxima aufweisen. Cowie (1985) ermittelte für eine Population von Theba pisana aus Wales eine obere Letaltemperatur von $42^{\circ} \mathrm{C}$ (bei $8 \mathrm{~h}$ Exposition) bis $46^{\circ} \mathrm{C}$ (bei $1 \mathrm{~h}$ ), für eine spanische Population $46-50^{\circ} \mathrm{C}$ Umgebungstemperatur. Für eine Population aus Australien waren $3 \mathrm{~h}$ bei $55^{\circ} \mathrm{C}$ letal (Kempster und Charwa 2003). In Arbeiten der Arbeitsgruppe der Autorin konnte gezeigt werden, dass bei verschiedenen Heideschneckenarten aus Südfrankreich die Temperatur, bei der 50\% Mortalität auftrat $\left(\mathrm{LT}_{50}\right)$, artspezifisch schwankte. Für Theba pisana lag die $\mathrm{LT}_{50}$ bei $41,5^{\circ} \mathrm{C}$, für Cernuella virgata bei $46,5^{\circ} \mathrm{C}$ und für zwei Populationen von Xeropicta derbentina bei $48-51{ }^{\circ} \mathrm{C}$ (Dittbrenner et al. im Druck). Diese artspezifische Hitzetoleranz spiegelt sich in der unterschiedlichen Proliferationsfähigkeit von Kalkzellen der Mittedarmdrüsen bei o. g. Arten wider. Es konnte gezeigt werden, dass die vergleichsweise temperatursensitive Population von Theba pisana im Gegensatz zu den sehr thermotoleranten Arten (Cernuella virgata, Xeropicta derbentina) nicht über die Möglichkeit verfügt, innerhalb kurzer Zeit den Flächenanteil an Kalkzellen in der Mitteldarmdrüse drastisch zu steigern (Dittbrenner et al. im Druck). Für die Kalkzellen in der Mitteldarmdrüse von Schnecken ist bekannt, dass sie eine wichtige Funktion bei der Aufrecht- 
erhaltung des Säure-Base-Gleichgewichts (Burton 1976) und bei der Osmoregulation (Taieb und Vicente 1999) spielen. Beide Prozesse werden bekanntermaßen durch hohe Temperaturen negativ beeinflusst (Heisler 1986). Bei den untersuchten Populationen konnte bezüglich der Induktion von Stressproteinen (syn. Hitzeschockproteinen) gezeigt werden, dass unterschiedliche Strategien verfolgt werden: Vor allem Xeropicta derbentina (in geringerem Maße auch Theba pisana) konnte bereits bei umweltrelevanten Temperaturen ihren Hsp70-Level signifikant erhöhen, während dies bei Cernuella virgata trotz hoher Hitzetoleranz nicht beobachtet werden konnte (Köhler et al. 2009). In dieser Studie korrelierten die maximalen Stressproteinlevel sehr gut mit der Variation im Schalenmuster, was als Hinweis auf epigenetische Regulation der Ausprägung des Schalenmusters durch Stressproteine interpretiert werden kann (Köhler et al. im Druck). In weiterführenden Studien soll diese Hypothese überprüft und die unterschiedlichen Anpassungsstrategien verschiedener Heideschneckenarten auf zellulärer und biochemischer Ebene zur Bewältigung von Hitzestress ausführlicher untersucht werden.

\section{Schlussfolgerungen}

Die dargestellten Einsatzmöglichkeiten von Gastropoden in der ökophysiologischen und ökotoxikologischen Forschung sollen verdeutlichen, dass Vertreter dieser Tiergruppe sehr gut als Modelle verwendet werden können, um Wirkungen von Umweltchemikalien und anderen Umweltstressoren darzustellen. Je nach Zielrichtung der Untersuchungen dienen Biomarkerantworten bei Schnecken entweder als Werkzeuge, um die Effektivität von Pflanzenschutzmitteln zu überprüfen sowie deren zelluläre Targets kennen zu lernen, oder aber dazu, nachteilige Effekte von Umweltstressoren zu beschreiben. Hierbei sind die gewonnenen Erkenntnisse nicht nur für den angewandten Umwelt- und Pflanzenschutz von Bedeutung, sondern sie ermöglichen auch im Sinne der Grundlagenforschung Prinzipien der Stressantwort $\mathrm{zu}$ erkennen und zu verstehen. Während im terrestrischen Bereich ein standardisierter Test mit Landschnecken für die Risikobewertung zur Verfügung steht, haben sich selbst sehr viel versprechende Testsysteme mit Schnecken in der aquatischen Ökotoxikologie bislang nicht dahingehend durchgesetzt, dass sie als standardisierte Tests akzeptiert worden wären. Derartiges wäre für die Zukunft jedoch äußerst wünschenswert.

\section{Literatur}

Allgaier C, Albert R (2008) Schadschnecken. Biologie, Arten und Bekämpfung. AID-Infodienst: $61 \mathrm{pp}$

Arad Z, Goldenberg S, Heller J (1993) Intraspecific variation in resistance to desiccation and climatic gradients in the distributi- on of the bush-dwelling land snail Trochoidea simulata. J Zool 229:249-265

Arad Z, Avivi TR (1998) Ontogeny of resistance to desiccation in the bush-dwelling snail Theba pisana (Helicidae). J Zool 244: $515-526$

Asami T (1993) Interspecific differences in desiccation tolerance of juvenile land snails. Funct Ecol 7:571-577

Barreiro R, Gonzalez R, Quintela M, Ruiz JM (2001) Imposex, organotin bioaccumulation and sterility of female Nassarius reticulatus in polluted areas of NW Spain. Mar Ecol Progr Ser 218:203-212

Berger B, Dallinger R (1993) terrestrial snails as quantitative indicators of environmental metal pollution. Env Monit Ass 25:65-84

Burton RF (1976) Calcium metabolism and acid-base balance in Helix pomatia. In: Davies S (ed), Perspectives of Experimental Biology vol. 1, Zoology. Pergamon Press, Oxford: p 7-16

Chabicovsky M, Klepal W, Dallinger R (2004) Mechanisms of cadmium toxicity in terrestrial pulmonates: programmed cell death and metallothionein overload. Environ Toxicol Chem 23(3):648-655

Chapman AD (2006) Numbers of Living Species in Australia and the World. Canberra: Australian Biological Resources Study. ISBN (printed) 978064256849 6; ISBN (online) 978064256850 2; $61 \mathrm{pp}$

Coeurdassier M, Gomot-de Vaufleury A, Lovy C, Badot PM (2002). Is the cadmium uptake from soil important in bioaccumulation and toxic effects for snails? Ecotox Environ Saf 53:425-31

Coughtrey TPJ, Martin MH (1977) The uptake of lead, zinc, cadmium, and copper by the pulmonate mollusc, Helix aspersa Müller, and its relevance to the monitoring of heavy metal contamination of the environment. Oekologia 27(1):65-74

Cowie RH (1985) Microhabitat choice and high temperature tolerance in the land snail Theba pisana (Mollusca: Gastropoda). J Zool Lond (A) 207:201-211

Dallinger R (2007) Umwelttoxikologie im Spannungsfeld zwischen Grundlagenforschung und Anwendung: Das Beispiel der Metallothioneine als Biomarker. Umweltwiss Schadst Forsch 19(1):35-42

Dallinger R, Wieser W (1984) Molecular fractionation of $\mathrm{Zn}, \mathrm{Cu}, \mathrm{Cd}$, and $\mathrm{Pb}$ in the midgut gland of Helix pomatia L. Comp Biochem Physiol 79C(1):125-129

Dallinger R, Berger B, Hunziker P, Kägi JH (1997) Metallothionein in snail $\mathrm{Cd}$ and $\mathrm{Cu}$ metabolism. Nature 17(388):237-238

Dallinger R, Berger B, Triebskorn R, Köhler HR (2001) Soil Biology and Ecotoxicology. In: Barker GM (ed), Biology of Terrestrial Molluscs. CAB1 Publishing p 489-527

Dallinger R, Chabicovsky M, Hödl E, Prem C, Hunziker P, Manzl C (2005) Copper in Helix pomatia (Gastropoda) is regulated by one single cell type: differently responsive metal pools in rhogocytes. Am J Physiol 189(4):R1185-R1195

Dittbrenner N, Lazzara R, Koehler HR, Mazzia C, Capowiez Y, Triebskorn R (im Druck) Heat tolerance in Mediterranean land snails: histopathology after exposure to different temperature regimes. J Moll Stud, DOI: 10.1093/mollus/eyn033

EU (2008) Updated risk assessment of 4,4'-isopropylidenediphenol (bisphenol-A). Draft environment addendum of May 2007. Rapporteur: United Kingdom. Environment Agency, Wallingford (unpublished, document code R325_0705_env). Richtlinie 2000/60/EG

Fechter R, Falkner G (1989) Weichtiere. Steinbach Naturführer. Mosaik Verlag, München. ISBN: 013466; 287 pp

Forbes VE, Selck H, Palmqvist A, Aufderheide J, Warbritton R, Pounds N, Thompson R, van der Hoeven N, Caspers N (2007a). Does bisphenol a induce superfeminization in Marisa cornuarietis? Part I: Intra- and inter-laboratory variability in test endpoints. Ecotox Environ Saf 66:309-318

Forbes VE, Aufderheide J, Warbritton R, van der Hoeven N, Caspers N (2007b) Does bisphenol a induce superfeminization in Marisa 
cornuarietis? Part II: Toxicity test results and requirements for statistical power analyses. Ecotox Environ Saf 66:319-325

Forbes VE, Warbritton R, Aufderheide J, van der Hoeven N, Caspers N (2008) Effects of bisphenol on fecundity, egg hatchability, and juvenile growth of Marisa cornuarietis. Environ Toxicol Chem 27(11):2232-2240

Gebhardt-Dunkel E (1953) Die Trockenresistenz bei Gehäuseschnecken. Zool Jb 64:235-266

Gibbs PE (2008) Long-term tributyltin (TBT)-induced sterilization of neogastropods: persistence of effects in Ocenebra erinacea over 20 years in the vicinity of Falmouth (Cornwall, UK). J Mar Biol Ass UK, DOI: 10.1017/S0025315408002336

Glamm N (2005) Papua Neuguinea: Erlebnisbericht Rabaul-Kavieng. 281 Seiten Pro Business-Verlag; ISBN-10: 3938262249; ISBN13: 978-3938262245, 281pp

Gomot A (1998) Toxic effects of cadmium on reproduction, development, and hatching in the freshwater snail Lymnaea stagnalis for water quality monitoring. Ecotox Environ Saf 41(3):288-297

Gomot-De Vaufleury A, Pihan F (2002) Methods for toxicity assessment of contaminated soil by oral or dermal uptake in land snails: metal bioavailability and bioaccumulation. Environ Toxicol Chem 21:820-827

Harmer SF (1895). The Cambridge Natural Story Vol. III. Obscure Press, Cambridge

Hartmann M, Weipert J (eds) (2006) Biodiversität und Naturausstattung im Himalaya II.-Verein der Freunde und Förderer des Naturkundemuseums Erfurt e. V., Erfurt, ISBN 10: 3-00-019541-6; ISBN 13: 978-3-00-019541-9, 524 pp

Heisler N (1986) Acid-base regulation in animals. Amsterdam: Elsevier Science Publishers

Henderson IF, Triebskorn R (2002) Molluscicidal chemicals. In: Barker GM (ed), Molluscs as crop pests. CABl Publishing, pp 1-32

Hispard F, Schuler D, de Vaufleury A, Scheifler R, Badot PM, Dallingen $R$ (2008) Metal distribution and metallothionein induction after cadmium exposure in the terrestrial snail Helix aspersa (Gastropoda, Pulmonata). Environ Toxicol Chem 27(7):1533-1542

Hollingsworth RG, Armstrong JW, Campbell E (2002) Pest Control: Caffeine as a repellent for slugs and snails. Nature 417:915916

Horiguchi T, Hyeon-Seo C, Shiraishi H, Shibata Y, Soma M, Morita M, Shimizu M (1998) Field studies on imposex and organotin accumulation in the rock shell, Thais clavigera, from the Seto Inland Sea and the Sanriku region, Japan Sci Tot Environ 214(1-3):65-70

ISO 15952 (2006) Soil quality-effects of pollutants on juvenile land snails (Helicidae)-Determination of the effects on growth by soil contamination

Kempster V, Charwa S (2003) Soil surface temperature and mortality in land snails: Implications for successful management. Austral J Exp Agricult 43:1351-1356

Khangarot BS, Ray PK (1988) Sensitivity of Freshwater Pulmonate Snails, Lymnaea luteola L. to Heavy Metals. Bull Environ Contam Toxicol 41:208-213

Knigge T, Mann N, Parveen Z, Perry C, Gernhöfer M, Triebskorn R, Köhler HR, Connock M (2002) Mannosomes: a molluscan intracellular tubular membrane system related to heavy metal stress? Comp Biochem Physiol C 131:259-269

Köhler HR, Triebskorn R (1998) Assessment of the cytotoxic impact of heavy metals on soil invertebrates using a protocol integrating qualitative and quantitative components. Biomarkers 3(2):109-127

Köhler HR, Triebskorn R (2004) Stress im Boden. Früherkennung ökotoxikologischer Effekte durch Biomarker. Biologie in unserer Zeit 34:240-248

Köhler HR, Rahman B, Gräff S, Berkus M, Triebskorn R (1996) Expression of the stress-70 protein family (hsp 70) due to heavy metal contamination in the slug Deroceras reticulatum: an approach to monitor proteotoxic stress conditions. Chemosphere 33(7):1327-1340

Köhler H-R, Belitz B, Eckwert H, Adam R, Rahman B, Trontelj P (1998) Validation of hsp70 stress gene expression as a marker of metal effects in Deroceras reticulatum (Pulmonata): Correlation with demographic parameters. Environ Toxicol Chem 17:2246-2253

Köhler HR, Kloas W, Schirling M, Lutz I, Reye AL, Langen JS, Triebskorn R, Nagel R, Schönfelder G (2007) Sex steroid receptor evolution and signalling in aquatic invertebrates. Ecotoxicology 16(1):131-143

Köhler HR, Lazzara R, Dittbrenner N, Capowiez Y, Mazzia C, Triebskorn R (2009) Snail phenotypic variation and stress proteins: do different heat response strategies contribute to Waddington's widget in field populations? J Exp Zool (Mol Dev Evol) 312B:136-147

Laguerre C, Sanchez-Hernandez JC, Köhler HR, Triebskorn R, Capowiez Y, Rault M, Mazzia C (im Druck). B-type esterases in the snail Xeropicta derbentina: an enzymological analysis to evaluate their use as biomarkers of pesticide exposure. Env Poll, DOI: 10.1016/j.envpol.2008.07.003

Marigomez I, Soto M, Kortabitarte M (1997) Tissue-level biomarkers and biological effect of mercury on sentinel slugs, Arion ater. Arch Environ Contamin Toxicol 31(1):54-62

Matthiessen P (2008) An assessment of endocrine disruption in molluscs and the potential for developing internationally standardized mollusc life cycle test guidelines. Integr Environ Ass Man 4(3):274-284

Matthiessen P, Gibbs PE (1998) Critical appraisal of the evidence for tributyltin-mediated endocrine disruption in molluscs. Environ Toxicol Chem 17(1):37-43

Mazurová E, Hilscherová K, Jálová V, Köhler HR, Triebskorn R, Giesy JP, Bláha L (2008). Endocrine effects of contaminated sediments on the freshwater snail Potamopyrgus antipodarum in vivo and in the cell bioassays in vitro. Aquat Toxicol 89:172-179

Oehlmann J, Schulte-Oehlmann U (2002) Molluscs as bioindicators. In: Markert BA, Breure AM, Zechmeister GH (eds), Bioindicators and biomonitors. Elsevier, pp 577-635

Oehlmann J, Schulte-Oehlmann U (2003) Endocrine disruption in invertebrates. Pure Appl Chem 75:2207-2218

Oehlmann J, Schulte-Oehlmann U, Fioroni P, Stroben E (1994) Probable extinction of prosobranch populations as result of TBT pollution in the Bay of Morlaix. Cah Biol Mar 35:255-256

Oehlmann J, Bauer B, Minchin D, Schulte-Oehlmann U, Fioroni P, Markert B (1998) Imposex in Nucella lapillus and intersex in Littorina littorea: interspecific comparison of two TBT-Induced effects and their geographical uniformity. Hydrobiologia 378:199-213

Oehlmann J, Schulte-Oehlmann U, Tillmann M, Markert B (2000) Effects of endocrine disruptors on prosobranch snails (Mollusca: Gastropoda) in the laboratory. Part I: bisphenol A and octylphenol as xeno-estrogens. Ecotoxicology 9:383-397

Oehlmann J, Schulte-Oehlmann U, Bachmann J, Oetken M, Lutz I, Kloas W, Ternes TA (2006) Bisphenol A induces superfeminization in the ramshorn snail Marisa cornuarietis (Gastropoda: prosobranchia) at environmentally relevant concentrations. Environ Health Perspect 114(Suppl 1):127-133

Oehlmann J, Di Benedetto P, Tillmann M, Duft M, Oetken M, Schulte-Oehlmann U (2007) Endocrine disruption in prosobranch molluscs: Evidence and ecological relevance. Ecotoxicology 16(1):29-43

Oehlmann J, Oetken M, Schulte-Oehlmann U (2008) A critical evaluation of the environmental risk assessment for plasticizers in the freshwater environment in Europe, with special emphasis on bisphenol A and endocrine disruption. Environ Res 108:140-149 
Osterauer R, Marschner L, Haus N, Dieterich A, Gerberding M, Triebskorn R, Betz O, Sures B, Köhler HR (2008) Effects of platinum group elements (PGE) on the embryonic development of the ramshorn snail Marisa cornuarietis and the zebrafish Danio rerio. Abstract for the YES-Meeting (Young Environmental Scientists Meeting), 16.-18.3.2009, Landau, Germany

Recio A, Marigomez JA, Angulo E, Moja J (1988) Zinc treatment of the digestive gland of the slug Arion ater L. 1. Cellular distribution of zinc and calcium. Bull Environ Contamin Toxicol 41:858-864

Reed-Judkins DR, Farris JF, Cherry DS, Heath AG, Cairns J Jr (1997) Functional responses in Leptoxis praerosa to increasing metal concentration and exposure duration. Environ Toxicol Chem 16(8):1666-1676

Regoli F, Gorbi S, Fattorini D, Tedesco S, Notti A, Machella N, Bocchetti R, Benedetti M, Piva F (2006). Use of the land snail Helix aspersa as sentinel organism for monitoring ecotoxicologic effects of urban pollution: an integrated approach. Env Health Perspect 114(1):63-69

Russel LK, Haven de JJ, Botts RP (1981) Toxic effects of cadmium on the garden snail (Helix aspersa). Bull Environ Contam Toxicol 26:634-640

Sawasdee B, Köhler HR (2008) The embryonic development of the ramshorn snail Marisa cornuarietis (Prosobranchia) is highly sensitive to metals. Abstract, StEvE meeting, Universität Tübingen, 28-29 November, 2008

Sawasdee B, Köhler HR (im Druck) Embryo toxicity of pesticides and heavy metals to the ramshorn snail, Marisa cornuarietis (Prosobranchia). Chemosphere, DOI: 10.1016/j.chemosphere.2009.01.085

Schirling M, Bohlen A, Triebskorn R, Köhler HR (2006) Establishment of an early life stage test for the apple snail Marisa cornuarietis to assess effects of potential endocrine disruptors. Chemosphere 64:1730-1738

Schmidt-Nielsen K, Taylor CR, Shkolnik A (1971) Desert snails: problems of heat, water and food. J Exp Biol 55:385-398

Schmitt C, Oetken M, Dittberner O, Wagner M, Oehlmann J (2008) Endocrine modulation and toxic effects of two commonly used UV screens on the aquatic invertebrates Potamopyrgus antipodarum and Lumbriculus variegatus. Environ Pollut 152:322-329

Schuder I, Port G, Bennison J (2003) Barriers, repellents and antifeedants for slug and snail control. Crop Protection 22(8):1033-1038

Schulte-Oehlmann U, Tillmann M, Casey D, Duft M, Markert B, Oehlmann J (2001) Östrogenartige Wirkungen von Bisphenol A auf Vorderkiemenschnecken (Mollusca: Gastropoda: Prosobranchia). Umweltwiss Schadst Forsch 13(6):319-333

Shachak M, Jones CG, Granot Y (1987) Herbivory in rocks and the weathering of a desert. Science 236:1098-1099

Sverdrup LE, De Vaufleury A, Hartnik T, Hagen SB, Loibnen AB, Jensen J (2006) Effects and uptake of polycyclic aromatic compounds in snails (Helix aspersa). Environ Toxicol Chem 25(7):1941-1945

Taieb N, Vicente N (1999) Histochemistry and ultrastructure of the crypt cells in the digestive gland of Aplysia punctata (Cuvier, 1803). J Moll Stud 65:385-398

Taylor MG (1995) Mechanisms of metal immobilization and transport in cells. In: Cajaraville MP (ed), Cell Biology in Environmental Toxicology. University of the Basque Country, pp $155-169$
Triebskorn R, Ebert D (1989) The importance of mucus production in slugs' reaction to molluscicides and the impact of molluscicides on the mucus producing system. Proc Brit Crop Prot Counc 41:373-379

Triebskorn R (2005) Biomarkers, Bioindicators, and the Trondheim Biomonitoring System. In: Lehr JH, Keeley J (eds), Water Encyclopedia: Water Quality and Resource Development. John Wiley and Sons, Inc., NJ, ISBN: 0-471-73686-4

Triebskorn R, Florschütz A (1993) Transport of uncontaminated and molluscicide-containing food in the digestive tract of the slug $D e-$ roceras reticulatum (Müller). J Moll Stud 59:35-42

Triebskorn R, Köhler HR (1992) Plasticity of the endoplasmic reticulum in three cell types of slugs poisoned by molluscicides. Protoplasma 169:120-129

Triebskorn R, Köhler HR (1996) The impact of heavy metals on the grey garden slug Deroceras reticulatum (Müller): Metal storage, cellular effects and semi-quantitative evaluation of metal toxicity. Env Poll 93:327-343

Triebskorn R, Köhler HR (2003) Cellular and molecular stress indicators as tools to assess effects and side-effects of chemicals in slugs. In: Slugs \& Snails: Agricultural, Veterinary and Environmental Perspectives. Brit Crop Prot Counc Symp Proc 80:69-76

Triebskorn R, Künast C (1990) Ultrastructural changes in the digestive system of Deroceras reticulatum (Mollusca, Gastropoda) induced by lethal and sublethal concentrations of the carbamate molluscicide Cloethocarb. Malacologia 32(1):87-104

Triebskorn R, Schweizer H (1990) Influence du molluscicide metaldehyde sur les mucocytes du tractus digestif de la petite limace grise (Deroceras reticulatum, Müller). ANPP-Deuxième Conference Internationale sur les Ravageurs en Agriculture, Versailles 3(1):183-190

Triebskorn R, Künast C, Huber R, Brem G (1990) The tracing of a 14C-labeled carbamate molluscicide through the digestive tract of Deroceras reticulatum. Pestic Sci 28:321-330

Triebskorn R, Köhler HR, Zahn T, Vogt G, Ludwig M, Rumpf S, Kratzmann M, Alberti G, Storch V (1991) Invertebrate cells as targets for hazardous substances. Z Angew Zool 78(3):277-287

Triebskorn R, Henderson IF, Martin A, Köhler HR (1996) Slugs as target and non-target organisms for environmental pollution. Brit Crop Prot Counc 66:65-72

Triebskorn R, Christensen K, Heim I (1998) Effects of orally and dermally applied metaldehyde on mucus cells of slugs (Deroceras reticulatum) depending on temperature and duration of exposure. J Moll Stud 64:355-375

Triebskorn R, Henderson IF, Martin AP (1999) Detection of iron in slugs (Deroceras reticulatum) contaminated with iron-chelates by means of energy filtering transmission electron microscopy (EFTEM). Pest Sci 55:55-61

Triebskorn R, Bader K, Linder B, Köhler HR (2005). Snails and slugs as non-targets for environmental chemicals. IOBC Bull 28(6):1-9

Van der Hoeven N (2005) Experiments on the effect of BPA on the snail species Marisa cornuarietis as described in three papers by Oehlmann: Evaluation of the applied statistics and analysis of the raw data. ECOSTAT Report 05/011. 14 October 2005

Zaldibar B, Cancio I, Soto M, Marigomez I (2007) Digestive cell turnover in digestive gland epithelium of slugs experimentally exposed to a mixture of cadmium and kerosene. Chemosphere $70: 144-154$ 